\title{
EDITORIAL ARTICLE TITLE:
}

\section{Electronic consultations: A new art in clinical communication?}

\section{Author Information:}

$1^{\text {st }}$ author: Dr Faye Gishen, BSc MBBS FRCP SFHEA

University College London Medical School

Email: f.gishen@ucl.ac.uk

$2^{\text {nd }}$ Author and Corresponding author: Dr Naomi Gostelow. iBSc MBBS PGCert FHEA University College London Medical School

Address: Rm 138 Medical School Building, 74 Huntley Street, London, WC1E 6BT

Email: n.gostelow@ucl.ac.uk

Tel: 07762418437

Authorship statement: both authors fulfil the International Committee of Medical Journal Editors' criteria for authorship.

Disclaimer: the views submitted in this article are the views of the authors and not an official position of the institutions they are affiliated with.

Source(s) of support: no grants, funding or equipment, either internal or external were required in the production of this article.

Word Count: 1461 
Number of figures: 0

Number of boxes/tables: 2

References: 25

Conflict of interest: We have read the International Committee of Medical Journal Editors guidance on conflict of interest and declare we have no competing interests Funding: No funding, internal or external, was required for the production of this article Ethical approval: as a review article requiring no data collection or participants, ethical approval was not required.

Author contributions: The idea was conceived and originally drafted by Faye Gishen. Re-drafting of the work and addition of further intellectual content was done by Naomi Gostelow. Both authors took part in revising the work and gave final approval for its submission. Both authors agree to be accountable for all aspects of the work. 
Traditionally, doctors have relied on all of their senses to diagnose and treat patients; detecting subtle changes in body language, actively listening, and applying their professional wisdom and experience to clinical cases. This traditional model may be threatened by the increasing use of electronic consultations ('e-consultations'). This editorial focuses solely on the use of emails to conduct electronic consultations and without considering other models, such as Skype or video-conferencing. The authors consider the potential advantages and disadvantages of e-consultations for doctors, patients, health services and society.

The concept of the electronic consultation is barely a decade old and is evolving [1]. A meta-analysis conducted in 2016 by Liddy et al was unable to confidently define the prevalence of their use in the United Kingdom, but concluded that e-consultations are becoming increasingly utilised globally, particularly in the United States [2]. The explosion of information and digital technology combined with increasingly busy lifestyles has led to the quest for convenience in accessing professional services. In a US poll, $90 \%$ of respondents wished to communicate with their physician electronically[3] demonstrating a public perception that email communication offers quick and effective access to healthcare[4]. E-consultations are also increasingly being adopted by other healthcare disciplines, including nursing [5]. 
We find ourselves in a rapidly changing healthcare climate driven by an ageing population, increasing survival and life expectancy, and growing clinical complexity. Coupled with challenges to service funding, alternative cost and time-saving clinical measures must be sought. E-consultations could provide part of a solution to the growing volume of clinical work. E-consultations can be used to take a clinical history, diagnose, reassure and treat[6]. Some uses are contentious however, such as using them as part of a therapeutic treatment algorithm, leading to the remote prescribing of medications. This has been identified as a key concern for physicians conducting online consultations in addition to its appropriateness for complex cases, medico-legal aspects and logistical difficulty[7, 8].The General Medical Council (GMC), the United Kingdom's medical regulator, issued guidance on conducting remote consultations in response to this change in consultation style [9] with particular emphasis on remote prescribing; see Box 1 [10]. 
...remote prescribing should not be a matter of routine and should only be done if you have adequate knowledge of the patient's health and are satisfied that the medicine serves the patient's needs.

You are also expected to consider the limitations of communicating with a patient via the telephone or other technology; whether a physical examination is required and whether you have access to the patient's medical records.

Box 1. General Medical Council, 2012

As clinicians, we recognise that the application of emotional intelligence $[11,12]$ and career experience may be equally or more important than purely scientific interpretations of clinical presentations. However, by conducting consultations online doctors may become 'blinded' to the subtleties and nuances which are central in face-to-face consultations. Words in an email, without visual cues, risk being misinterpreted. There is a perception that the widespread adoption of the electronic consultation could erode the essence of the doctor-patient relationship and even reduce job satisfaction [8]. Some feel it could devalue the personal nature of the conventional therapeutic interaction and have labelled it a 'disruptive innovation'[13] carrying with it a risk of de-professionalisation[14], perhaps even leading to dilution in the quality of care. 
The counter argument is that e-consultations are practical and cost-saving, although this has not yet been definitively demonstrated[2]. In a climate of convenience and patientcentred care, the electronic consultation may save time and enable flexibility. Efficiency may be in part traded for effectiveness. One advantage that is becoming apparent is that remote consultations may allow the revealing of sensitive topics which may be too challenging for some patients to disclose face-to-face. For example, certain ethnic groups who have historically avoided presenting to doctors, may consider the e-consultation a less threatening or less stigmatising opportunity to be reviewed[15] . Similarly other geographically hard to reach populations may benefit from having electronic access to doctors [16]. This may have wider applications such as within the prison population, visiting whom has long been a time-consuming activity, and to which e-consultations could offer a pragmatic compromise.

Groups less familiar with technology, such as the elderly, could suffer as e-consultations become more prevalent. Also disadvantaged could be those who cannot afford or do not own a smart phone or computer. In addition, good internet access is not ubiquitous, potentially introducing differences in availability. The situation may also further social and health inequalities amongst those with learning disabilities or illiterate individuals and these observations are reflected in the concerns of primary care physicians working in diverse communities [8]. When advocating emails as a consultation option, doctors 
should consider the suitability of the patient's condition, their technological ability and compliance[17].

A further concern is the potential contribution of e-consultations to professional fatigue in doctors. Many already experience 'compassion fatigue'[18,19] and are at risk of 'burnout' $[20,21]$. Some practitioners are unable to effectively detach themselves from clinical and administrative responsibilities. The legacy of more flexible and convenient services is rising patient expectation. One study showed patients with e-mail access to their doctor expected response times of less than 24 hours for routine questions or results[22]. The threat of requiring continuous access to emails, may further test doctors' vitality and resilience. Within certain healthcare systems there are also questions of how remote consulting should be renumerated if not confined to the surgery or normal working day [23]. Conversely, as electronic communication may allow flexibility in practice, with doctors and patients not being geographically constrained to particular sites, this may actually confer lifestyle advantages as well as lower infrastructural costs.

Providing that good governance is understood and achievable, and there is scrupulous recording of e-consultations with the ability to share communications within the multidisciplinary team (MDT) in a timely way, this could offer a viable adjunct to face-to-face clinical work. Electronic backup systems are also vital in order to ensure safety. In reality, 
using electronic consultations to replace some traditional consultations may become a modern professional necessity. Furthermore, e-consultations could embody the collaborative approach between doctors and patients with greater autonomy afforded to patients [24].

This type of innovation also facilitates movement towards the global workplace. Communities of practice now extend beyond geographical and sociological borders, largely enabled by advances in information technology and a more global ethos in healthcare. In large countries with remote and rural communities, such as Australia, E-consultations have successfully been used, not only for patient interactions, but for consultations and advice between specialties, improving access and knowledge sharing[15, 23].

Doctors have expressed concerns regarding the use of emails for inappropriate clinical situations, including emergencies[7]. This concern is reflected in guidance from regulating bodies advising clinicians obtain informed consent from patients regarding the benefits and limitations of e-consultations[26, 17]. Guidance from both US and UK bodies advises that legal, ethical and professional rules are still applicable when communicating with patients via email and there is a general consensus this is best reserved for stable patients with chronic conditions[7].Table 1 summarises this guidance. [Table 1 near here]. 
- Some conditions are unsuitable for remote consultations because of the inability to conduct a physical examination, to monitor treatment and to provide appropriate aftercare.

- Doctors have an ethical and legal duty to ensure that personal data and communications systems are secure

- Doctors must ensure that patients understand the limitations of online consultations and give informed consent

- Doctors should only prescribe if they have enough information about the patient, and sufficient dialogue with the patient to do so safely

Medical Defence Union, 2013
- Online communications are best suited for patients previously seen and evaluated in an office setting

- Clinicians should discourage the use of online communication to address medical emergencies and instead instruct patients to go to the emergency department

- Informed consent should be obtained from the patient regarding the appropriate use and limitations of this form of communication

- Clinicians should exercise discretion when selecting patients for the use of online services to ensure they are capable of using electronic communication

Medem eRisk Working Group for Healthcare, 2006 
Preparing future doctors for $21^{\text {st }}$ century challenges means addressing these issues in their training, acknowledging the benefits and limitations and medico-legal and ethical pitfalls. Doctors in training need to learn how such new technologies can be used alongside traditional ones to augment communication skills and clinical reasoning. This may need to be incorporated into future medical curricula.

In summary, the changes discussed pose both a threat and an opportunity. This represents a paradigm shift in communication with a change in the classical consultation model. The associated reduction in non-verbal cues, means that practitioners can no longer solely rely on their traditional clinical tools and training and need to acquire new skills to adapt to and manage these changes. This new model of consultation enables previously elusive, doctor-fearing groups access to physicians and offers flexibility, and financial implications which may be significant and welcome. However, safeguards need to be put in place to ensure the safety and accountability of electronic consultations. We pose the question of how doctors are going to lead the way in preventing erosion of professionalism in this context?

We acknowledge that this editorial is limited by choosing email consultations as a focus and remote consultations may take place via other, more contemporary media such as video-conferencing or apps. However, by choosing to discuss arguably the most 
established form of remote consultations we have highlighted that, despite no longer being considered technologically advanced, email consultations are still clouded by uncertainty with regards to what they can be used for, medico-legal and professional issues. It is vital that the increasing prevalence and demand for remote consulting does not outstrip professional experience or guidance.

Turbulence and discomfort is to be expected with professional shifts. Doctors and those professionally allied to medicine, are beginning to appreciate and evaluate the depth, threat and implications of the electronic consultation on their professional and personal lives. It is important that doctors take a leading role in innovation, ensuring that it is what patients want whilst protecting the confidential relationship, promoting health equity and above all, ensuring safety. The remote electronic consultation may offer an opportunity for evolution in the art of medicine; a new art in clinical communication.

\section{Bibliography}

1. Aas, I. Organising for remote consultations in health care- the production process. Behaviour \& Information Technology. 2003;22 (2), p.91-100. 
2. Liddy, C. Electronic Consultation Systems: Worldwide Prevalence and their Impact on Patient Care- A Systemic Review. Fam. Pract. 2016;33(3):274-285.

3. Couchman, G. Forjuoh, S, Rascoe, T. Email communications in family practice:what do patients expect? J Fam Pract. 2001; 50 (5):414-8

4. Car, J and Sheikh, A. Email consultations in health care:2 acceptibility and safe application. BMJ. 2004:329-439. doi: https://doi.org/10.1136/bmj.329.7463.439

5. Royal College of Nursing (2012). Using technology to complement nursing practice; an RCN guide for health care practitioners

6. Pinnock, H. Triage and remote consultations: Moving beyond the rhetoric of access and choice. $\mathrm{Br} J$ Gen Pract. 2005;55(521):910-91.doi: 10.3399/bjgp16X684001

7. Patt, M. Houston, T. Jenckes, M. et al. Doctors who are using email with their patients: a qualitative exploration. J med Internet Res. 2003; 5(2):e9. doi:10.2196/jmir.5.2.e9

8. Hanna L. May C. Fairhurst K. The place of information and communication technology-mediated consultations in primary care: GPs' perspectives. Family Practice. 2012; 29: 361-66.DOI: 10.1093/fampra/cmr087 
9. General Medical Council (GMC). Good Medical Practice. 2013. Available from: http://www.gmc-uk.org/guidance/good medical practice/duties of a doctor.asp [accessed 05/2017].

10. General Medical Council Remote patient consultations and prescribing in Good Medical Practice. 2013. Available at: http://www.gmc-uk.org/guidance/30549.asp [accessed 05/2017]

11. Russell, C. Exploring how emotionally intelligent leaders apply related competencies to manage complex challenges. 2014. ProQuest Dissertations Publishing. Available at: http://gradworks.umi.com/36/21/3621797.html [accessed 05/2017]

12. Goleman, G. Emotional Intelligence: why it can matter more than IQ. London: Bloomsbury. 1996

13. Gupte, G. Vimalananda V, Simon S. et al. Disruptive Innovation: Implementation of Electronic Consultations in a Veterans Affairs Health Care System. JMIR Med Inform 2016;4(1):e6 doi: 10.2196/medinform.4801

14. Haug, M. 'Deprofessionalisation: an alternate hypothesis for the future'.Soc Rev Monograph. 1973; 120:195-211.

15. Goldschmidt, R \& Graves, D. The National HIV Telephone Consultation Service (Warplane): a clinical resource for physicians caring for African-Americans. Journal of the National Medical Association. 2013:95(2) 
16. Byrom, L. et al. Tele-Derm national: a decade of teledermatology in rural and remote Australia. Aust J Rural Health. 2016;24(3):193-9 doi: 10.1111/ajr.12248

17. eRisk Working Group for Healthcare: guidelines for online communication. 2006. Available from: www.medem.com/phy/phy_eriskguidelines.cfm [accessed 05/2017]

18. Driver, D. Compassion fatigue: a threat to doctors' health. Medical Post. 1997;33 $(41): 77$

19. Johne,M. Compassion fatigue: a hazard of caring too much. Medical Post. 2006; $42(3): 19$

20. Amoafo, E. Hanbali, N. Patel, A et al. What are the significant factors associated with burnout in doctors? Occup Med. 2015;65(2):117-121 doi: 10.1093/oc$\mathrm{cmed} / \mathrm{kqu} 144$

21. Graham J, Potts, H. Ramirez, A. Stress and burnout in doctors. Lancet. 2002; 360(9349):1975-1976

22. Couchman, G. Forjuoh, S, Rascoe, T. Email communications in family practice:what do patients expect? J Fam Pract. 2001; 50 (5):414-8 
23. Hanna L. Fairhurst K. Using information and communication technologies to consult with patients in Victorian primary care. The views of general practitioners. Australian Journal of Primary Health. 2013; 19(2). 166-70.

24. Whitty, G. Changing modes of teacher professionalism: traditional, managerial, collaborative and democratic. In B. Cunningham (Ed.), Exploring Professionalism. 2008. London: Bedford Way Papers

25. North, F. Uthke, L. Sidna, M. et al. Integration of e-consultations into the outpatient care process at a tertiary medical centre. J Telemed Telecare. 2014;20(4):221-229 doi: $10.1177 / 1357633 \times 14533886$

26. Medical Defence Union. Remote Prescribing (2017). Available from: https://www.themdu.com/guidance-and-advice/guides/prescribing [accessed 05/2017] 